\title{
Multivariable relationships between autonomic nervous system related indices in hyperbaric environments
}

\author{
Cristina Pérez-Martínez ${ }^{1}$, María Dolores Peláez-Coca ${ }^{1,2}$, Alberto Hernando ${ }^{1,2}$, Eduardo Gil ${ }^{1}$, Carlos Sánchez ${ }^{1,2}$
}

\begin{abstract}
The main aim of this work is to model the relationships between parameters extracted from the heart rate variability (HRV) signal, which is derived from the electrocardiogram (ECG), at different stages of a simulated immersion in a hyperbaric chamber. The response of the Autonomic Nervous System is known to be affected by changes in atmospheric pressure, reflected in changes in the HRV signal. A dataset consisting of ECG signals from 17 subjects exposed to a controlled hyperbaric environment, simulating depths from 0 $\mathrm{m}$ to $40 \mathrm{~m}$, was used. Both linear and nonlinear dependences of HRV parameters were analysed using linear regression and Mutual Information (entropy-based) techniques. Furthermore, relationships between parameters of the HRV signals, biophysical variables of the subjects, and atmospheric pressure changes were characterized by artificial neural networks. In particular, self-organizing maps (SOM) were trained for modelling and clustering all the data. In the mid-term, these models could be the basis to create predictive models of HRV parameters at high depths in order to increase the safety for divers by warning them if some abnormal body response could be expected just by processing the ECG signal at sea level before immersion.
\end{abstract}

\section{INTRODUCTION}

Diving is currently one of the most demanding sport activities. The human body is affected by different factors in the aquatic environment, which require biological and physiological adaption to maintain homeostasis. A bad adaption to a hyperbaric environment could entail serious health problems, and thus the maximum depth for recreational diving is limited to $40 \mathrm{~m}$ (equivalent to a pressure of $5 \mathrm{~atm}$ ).

As stated by Poisseuille's law, a decrease in the heart rate is needed to maintain an adequate cardiac output and to minimise the effects of hydrostatic pressure [1]. The main factors affecting cardiovascular response during diving are a lower temperature and a higher pressure [2]. The Autonomic Nervous System (ANS) plays a key role in maintaining homoeostasis by controlling, among others, cardiac output, heart rate, and blood pressure. The balance between the two branches of the ANS, namely the sympathetic nervous system and the parasympathetic nervous system, reflects the efforts of the body to adapt to a new environment in the best way possible [3]. The analysis of the Heart Rate Variability signal (HRV), which can be directly derived from the electrocardiogram (ECG), is a commonly used non-invasive technique to measure the activity of the ANS. Spectral analysis of the HRV signal reveals two main components: a low-frequency (LF) component $[0.04-0.15 \mathrm{~Hz}$, which reflects both sympathetic and parasympathetic activity, and

\footnotetext{
${ }^{1}$ BSICoS group, Aragón Institute of Engineering Research (I3A), IISAragón, University of Zaragoza, Spain

${ }^{2}$ Centro Universitario de la Defensa (CUD), Zaragoza, Spain
}

a high-frequency (HF) component [0.15 - $0.4 \mathrm{~Hz}]$, which has been typically used to measure parasympathetic activity [3].

The ANS response has been analysed in some studies simulating variable atmospheric pressure conditions inside a hyperbaric chamber, without the need to go under water. The results of these studies suggested an increase in the power related to the HF band, thus meaning an increase in parasympathetic activity [4], [5]. A number of these studies observed a decrease in the heart rate [4], [6], [7], although this trend was not shown in some of them [5]. The number of studies analysing the response of the ANS during immersion is low, probably due to the implicit difficulty of obtaining proper signal recordings. In real dives, despite the fact that other external uncontrolled variables, such as immersion reflex or cold water stimulus [8], [9], might lead us to expect an increase in sympathetic activity, the ANS response shows a similar behaviour to that in a hyperbaric chamber: an increase in HF power [10], [11]. This pattern could be explained by a possible rise in baroreceptor sensitivity, with the corresponding increase in parasympathetic activity [12]. All these studies are characterised by: a small number of subjects (about 10), the use of only the HRV to characterise the ANS behaviour, and the measurement of only one highpressure stage between 2.5 and $3 \mathrm{~atm}$.

The main aim of this work is to model the relationships between parameters extracted from the HRV signals of 17 subjects at five different stages of a simulated immersion in a hyperbaric chamber: initial depth of $0 \mathrm{~m}$, depth of $20 \mathrm{~m}$ during descent, depth of $40 \mathrm{~m}$ after descent, depth of $20 \mathrm{~m}$ during ascent, and depth of $0 \mathrm{~m}$ after ascent. Both linear and nonlinear dependences are analysed by using linear regression and mutual information techniques. Furthermore, artificial neural networks (ANNs) were trained to identify multivariable relationships between parameters of HRV signals, biophysical variables of the subjects, and atmospheric pressure at different depths. Particularly, selforganizing maps (SOM) are calculated to help to detect these relationships by identifying clusters of variables. In the midterm, this analysis could serve as a basis to create more complex reliable predictive models of HRV parameters at high depths with the objective of increasing the safety for divers: if the estimated parameters obtained with the model for a particular subject (with ECG monitored before diving) were far from the physiological healthy range, thus indicating some possible abnormal body response, immersion could be disadvised. 


\section{RELATIONSHIPS BETWEEN VARIABLES}

\section{A. Materials}

ECG signals of 17 subjects (14 males and 3 females), with ages between 20 and 44 years, were used as database. The protocol inside the chamber had a duration of about two hours, and the ECG signal was recorded at five different stages, as described in [13]: resting at 1 atm (sea level), $B 1 D^{1}$, stop at $3 \mathrm{~atm}$ simulating a depth of $20 \mathrm{~m}, B 3 D$, stop at 5 atm simulating a depth of $40 \mathrm{~m}, B 5$, and subsequently two more stops during ascent at $3 \mathrm{~atm}$ and $1 \mathrm{~atm}$, stages $B 3 A$ and $B 1 A$, respectively.

Four temporal parameters were computed using the beatto-beat time series of the ECG signal, and four frequency parameters were calculated based on the power spectral distribution of the HRV signal [13]:

- $N N(s)$ : median of the Normal-to-Normal $(\mathrm{NN})$ intervals between the fiducial points.

- $\operatorname{SDNN}(s)$ : statistical dispersion of the NN intervals by measuring the interquartile range, which reflects the difference between the upper and lower quartiles.

- $R M S S D(s)$ : square root of the mean of the squares of the successive differences between adjacent $\mathrm{NN}$ intervals.

- $p N N 50(\%)$ : number of pairs of successive NNs that differ by more than $5 \mathrm{~ms}$, divided by the total number of $\mathrm{NN}$ intervals.

- $P_{L F}(a d)$ : power inside the LF band $(0.04-0.15 \mathrm{~Hz})$.

- $P_{H F}(a d)$ : power inside the HF band (0.15-0.4 Hz).

- $P_{L F n}(n u)$ : power in LF band normalised with respect to those of the LF and HF bands:

$$
P_{L F n}=\frac{P_{L F}}{P_{L F}+P_{H F}}
$$

- $R_{L F / H F}(n u)$ : ratio between $L F$ and HF power:

$$
R_{L F / H F}=\frac{P_{L F}}{P_{H F}}
$$

These eight parameters (denoted as indices in the rest of this paper) were computed at the five stages of immersion allowing proper characterization of the response of the ANS to both atmospheric pressure changes and time spent in the hyperbaric chamber. Additionally, biophysical information of each subject was included in the database.

\section{B. Linear Regression}

In order to analyse the linear dependence between pairs of variables, linear regression technique was used. If the value of the square of the Pearson's coefficient, $R^{2}$, was equal or greater than 0.75 , the relationship between the parameters was considered to be linear.

The results showed some punctual and minor linear relationships between indices at different stages. Only the temporal parameters NN and RMSSD at B1D stage showed a linear dependence on these same variables at other stages.

Similarly, the relationships between indices and biophysical variables showed high results at stage B5 between $P_{L F}$

\footnotetext{
${ }^{1} \mathrm{~B}$ from basal; the number reflects the pressure in atm; the letter $\mathrm{D}$ or $\mathrm{A}$ refers to descent or ascent
}

and if the subject takes any medication and also between $P_{L F}$ and if the subject drinks caffeine. In other words, ANS response at high pressure is notably affected by these two biophysical parameters.

\section{Mutual Information}

Mutual information (MI) measures how much one random variable tells us about another one. A high value of MI indicates that a variable can be predicted if the other one is known. On the contrary, if MI is close to zero, both variables can be considered independent on each other. Unfortunately, MI does not inform about the type of relationship, linear or nonlinear.

In this work, the normalized MI (nMI) within the $[0,1]$ interval was used. The nMI value is an indicator of how acceptable a model using a pair of variables could be. Analogously to linear regression, pairs of parameters were considered to be highly dependent on each other if their nMI was equal or greater than 0.75 .

The results of nMI showed a strong dependence between the NN and RMSSD parameters at all different stages, adding more information to that provided by linear regression. Finally, other relationships were observed in B3D, B5 and B1A stages. For example, some positive relationships between pNN50 index at B1D stage with NN, pNN50, $P_{L F n}$ and $R_{L F / H F}$ at B5 stage; $P_{L F}$ at B1D with NN, RMSSD, pNN50, $P_{L F}$ and $P_{H F}$ at B1A stage; and $P_{H F}$ at B1D stage with NN, RMSSD, pNN50 and $P_{H F}$ at B1A stage.

After performing a similar analysis between biophysical parameters and the indices at all stages, the most significant results were found for high arterial pressure value and cardiac rate with the indices of all stages, except for $P_{H F}$ in ascent stages. Also, arterial pressure values shared information with $\mathrm{NN}$ and pNN50 at 1 atm, while this biophysical parameter was correlated with both SDNN and $R_{L F / H F}$ when pressure was increased, i.e. at $5 \mathrm{~atm}$. On the other hand, isolated positive relationships were observed with age, height and low arterial pressure parameters. Low nMI values for low arterial pressure with $\mathrm{pNN} 50$ and $R_{L F / H F}$ at stage B3D, and SDNN at stage B1D were also observed (less than 0.4). In the rest of the relationships with biophysical parameters, nMI values were very low (between 0.1 and 0.4 ).

\section{SELF-ORGANIZING MAPS}

Artificial Neural Networks (ANNs) were used to detect any relationship between indices, biophysical variables, stages and subjects. Therefore, self-organizing maps (SOMs) were trained to detect possible multivariable relations between parameters. A default unsupervised training was chosen since the main goal was to clusterize the data: the training process was initialized in a random way, was linear and a batch training algorithm was selected. A Gaussian function was used as a neighborhood function for updating the weights of the neurons.

The principal quantifiable properties used to measure the reliability of a trained SOM were [14]: 
- Mean quantization error to represent the precision of the data.

- Topographic error to represent the precision of the topology.

\section{A. Self-organizing maps of the stages and indices}

The SOM input data were normalized in the interval $(0,1)$ with respect to the values at the B1D stage. The maps were $7 \times 7$ in size with hexagonal grids. This size was chosen based on the number of data for training. Smaller SOMs would lead different data vectors activating neighboring cells, whereas larger SOMs would probably lead to isolated activations for each data vector. The Umatrix (unified distance matrix) is a common representation of a SOM for data visualization.

The activation maps of the indices at each stage were calculated (Fig. 1). NN and RMSSD had a similar map in both $\mathrm{B} 3 \mathrm{D}$ and $\mathrm{B} 1 \mathrm{~A}$ stages, meaning a strong connections between these two parameters. In addition, $P_{L F n}$ and $R_{L F / H F}$ maps at B3D and B3A stages are very similar as well.

Interestingly, SOMs of each index were different at all stages (Fig. 2). Even though the pressures of B1D and $\mathrm{B} 1 \mathrm{~A}$ stages, and $\mathrm{B} 3 \mathrm{D}$ and $\mathrm{B} 3 \mathrm{~A}$ stages were the same, their activation maps did not share any similarity. Therefore, both time spent in a hyperbaric environment and being in a descent or ascent stage are factors that significantly affect the values of the indices.

\section{B. Self-organizing maps using the values of the indices}

Three cases of study were analysed:

- Case 1: the characteristics are the 8 temporal and frequency parameters, the samples are the data of every subject at each stage, and the map is labelled according to the stages. The mean quantization error is close to zero (see Table I), therefore this map is an acceptable result of classification. Although a low value of topographic error is convenient, this value is exactly zero, not guaranteeing a good organization of the map. Large dispersion of the four normalized stages was detected over the Umatrix, particularly, at B3D stage.

- Case 2: the characteristics are the 4 stages, the samples are the 8 indices of every subject, and the map is labelled according to the indices. Both errors have quality values close to zero (see Table I), so this classification is acceptable, even if no individual clusters can be detected. For example, NN parameter always shares activated nodes with other indices.

- Case 3: the characteristics are the 8 temporal and frequency parameters of the 4 stages, i.e. 32 characteristics in total, and the map is labelled according to the subjects. In this case, the size of the map was chosen to be $6 \times 6$, good enough to classify the 17 subjects. If the subjects had the same behaviour, all the activated nodes of the Umatrix would be in the same region. But, in a global view, subjects present high variability in their physiological ANS responses. A few subjects were found to be isolated within the maps, probably meaning their ANS responses significantly differ from those of the others.

TABLE I: Different error values of the three cases of study.

\begin{tabular}{cccc} 
& Case 1 & Case 2 & Case 3 \\
\hline Mean quantization error & 0.0981 & 0.0720 & 0.2278 \\
\hline Topographic error & 0 & 0.0956 & 0.0588 \\
\hline
\end{tabular}

\section{Self-organizing maps using the indices and biophysical features}

In order to use the biophysical features as inputs for the SOMs, they were previously normalized within the interval $(0,1)$. Some of the features, such as 'Alcohol' or 'Caffeine', took only binary values of 0 or 1 .

Activation maps of biophysical variables are shown in Fig. 3. Only the similarity between height and weight maps was observed. SOMs combining indices at B1D stage and biophysical parameters showed that low arterial pressure was not relevant for subject classification since the maps with and without that variable were practically the same. Besides, Umatrices of height and weight were similar, and the same occurred for those of high arterial pressure and pain.

\section{DISCUSSION}

In this study, the relationships between physiological parameters related to the activity of the ANS at different depths during simulated immersions have been assessed. In a longer term, the objective would be to develop a reliable predictive model to predict the risk of diving for a particular subject. The results of this work could serve as the initial step in that direction, for example reducing the number of variables needed, based on the multivariable relationships shown in the SOMs. However, for creating such a predictive model, the database should be considerably enlarged so that the training could lead to a valid model for all the parameters involved in the ANS adaptation to a hyperbaric environment.

The analysis of parameters at the different stages of immersion shows larger linear relationships between temporal indices than those between frequency ones. For instance, positive results are found between NN and RMSSD at B1D and B5 stages looking at $R^{2}$ values. Regarding nMI values, they are larger in general (at least 0.5 in most relationships) than those of $R^{2}$. Some discrepancies are also observed between $R^{2}$ and $\mathrm{nMI}$, which may be due to the mapping of some variables within the $[0,1]$ interval needed for calculating nMI. Nowadays, MI and nMI are being used in the biomedicine field in classification and approximation works. They help to select relevant characteristics within datasets and to reduce the computational complexity quantitatively [15].

The same statistical methods (linear regression and nMI) between stages including the biophysical features were performed. The results show that cardiac rate variable, measured with a pulsometer before immersion, is linearly related with NN and RMSSD, and $P_{L F}$ at B5 stage with if the subject takes any medication and if the subject drinks caffeine 


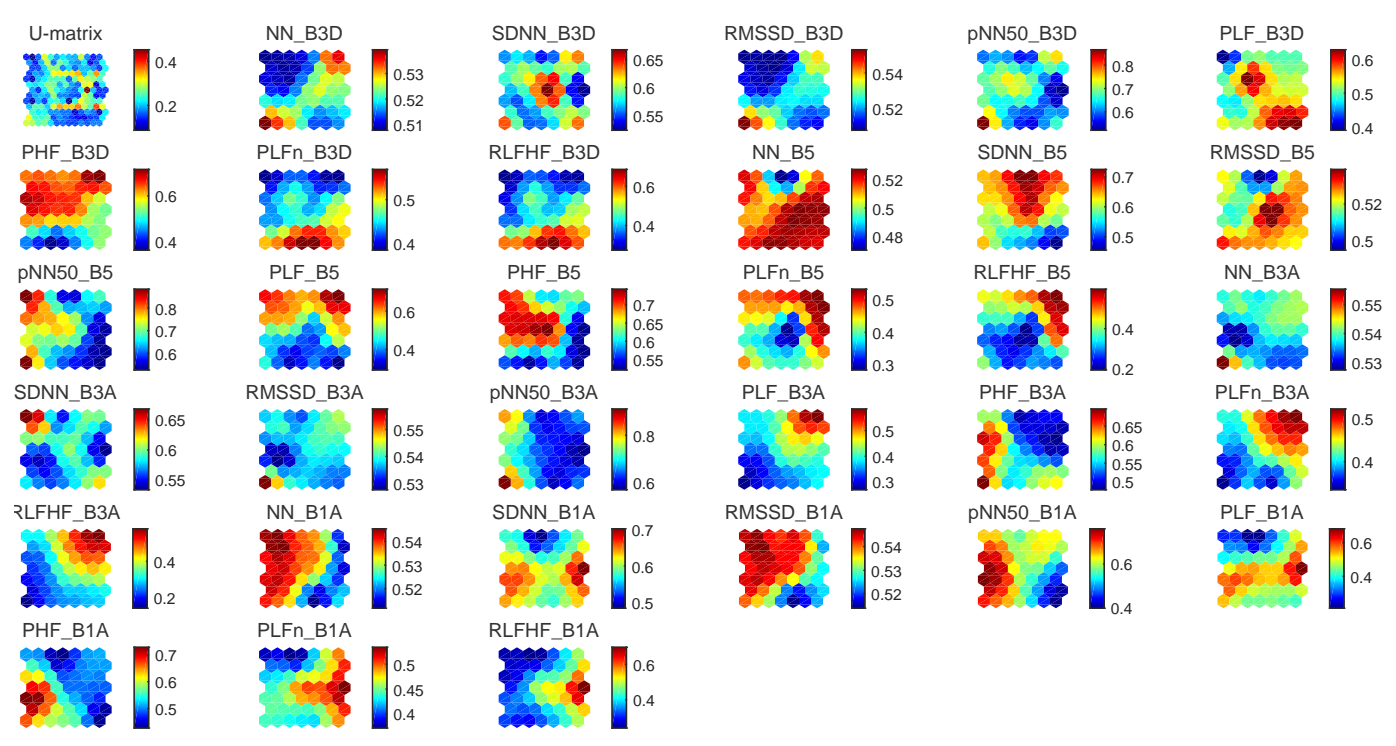

Fig. 1: Activation maps of the indices at each stage after training.

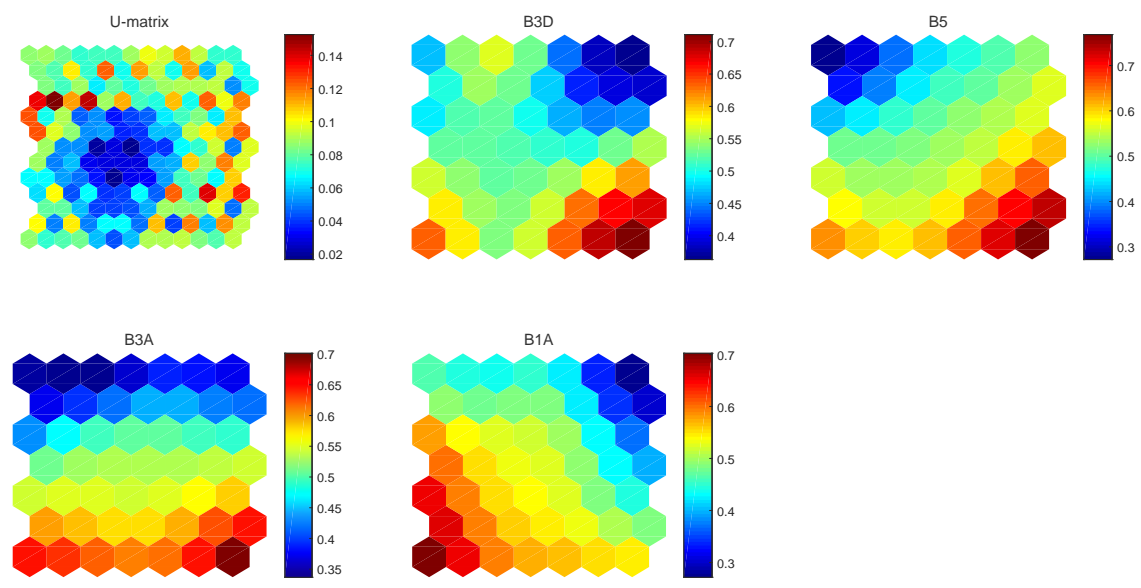

Fig. 2: Activation maps of the stages after training.

variables. In this case, there is a larger diversity of nMI values: insignificant relations are observed between if the subject drinks caffeine variable and the indices at B5 stage, but the high and low arterial pressure variables are highly related to those indices in terms of nMI. Interestingly, the if the subject practices diving variable and the indices at B5 stage present no dependence (nMI values equal to zero).

In the article [16], the influence of time and depth in the ANS response is confirmed, just as it was concluded with the SOMs showing organization of the stages. In the study by Kujawski et al. [17], the influence of the time spent within a hyperbaric environment on the cardiovascular system was also observed, registering changes in the parasympathetic branch at a depth of $30 \mathrm{~m}$, while both ANS branches were affected at a depth of $60 \mathrm{~m}$.

\section{CONCLUSIONS}

In this study, only a few parameters related to the ANS response in hyperbaric environment showed significant linear dependences between them, meaning the relationships are either nonlinear or multivariable. In order to account for nonlinear dependences, the results using Mutual Information show a considerably higher number of significant relationships.

Multivariable dependence was assessed using ANNs, in particular, SOMs. Both the time spent in a hyperbaric environment and if the subject is in an ascent or descent stage play a key role in the ANS response. Furthermore, both the observed high inter-subject variability in the ANS response and the high number of variables that can have a potential influence on this response make it difficult to create generic behavioral models.

\section{ACKNOWLEDGEMENTS}

This work has been financed by Ministerio de Economía, Industria y Competitividad and by funds FEDER (project RTI2018-097723-B-I00); by Centro Universitario de la Defensa (CUD) under the projects CUD2018-08 and 

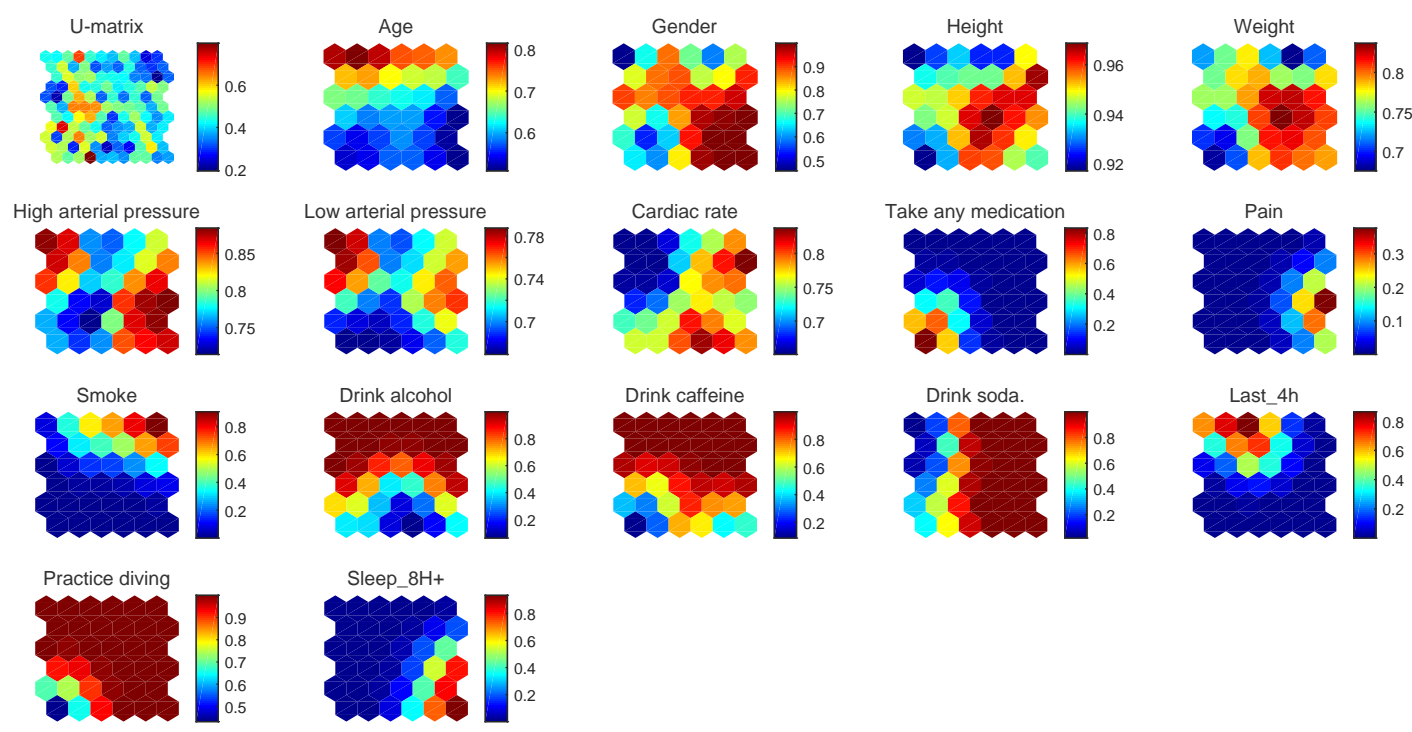

Fig. 3: Activation maps of the biophysical variables of the 17 subjects after training.

UZCUD2017-TEC-04; and by Gobierno de Aragón (Reference Group BSICoS T39-17R) cofunded by FEDER 20142020 "Building Europe from Aragon". This work was possible thanks to the collaboration with the Hospital General de la Defensa en Zaragoza and volunteers from the Regimiento de Pontoneros y Especialidades de Ingenieros no12.

\section{REFERENCES}

[1] E. P. Widmaier, H. Raff, and K. T. Strang, Vander's human physiology: the mechanisms of body function. McGraw-Hill Higher Education, 2008.

[2] A. Pelliccia, R. Fagard, H. H. Bjørnstad, A. Anastassakis, E. Arbustini, D. Assanelli, A. Biffi, M. Borjesson, F. Carrè, D. Corrado, et al., "Recommendations for competitive sports participation in athletes with cardiovascular diseasea consensus document from the study group of sports cardiology of the working group of cardiac rehabilitation and exercise physiology and the working group of myocardial and pericardial diseases of the european society of cardiology," European heart journal, vol. 26, no. 14, pp. 1422-1445, 2005.

[3] A. Camm, M. Malik, J. Bigger, G. Breithardt, S. Cerutti, R. Cohen, P. Coumel, E. Fallen, H. Kennedy, R. Kleiger, et al., "Heart rate variability: standards of measurement, physiological interpretation and clinical use. task force of the european society of cardiology and the north american society of pacing and electrophysiology," Circulation, vol. 93, no. 5, pp. 1043-1065, 1996.

[4] E. Barbosa, J. M. García-Manso, J. M. Martín-González, S. Sarmiento, F. J. Calderón, and M. E. Da Silva-Grigoletto, "Effect of hyperbaric pressure during scuba diving on autonomic modulation of the cardiac response: application of the continuous wavelet transform to the analysis of heart rate variability," Military medicine, vol. 175, no. 1, pp. 61-64, 2010.

[5] V. Lund, E. Kentala, H. Scheinin, J. Klossner, K. Sariola-Heinonen, and J. Jalonen, "Hyperbaric oxygen increases parasympathetic activity in professional divers." Acta physiologica Scandinavica, vol. 170, no. 1, pp. 39-44, 2000.

[6] K. Yamauchi, Y. Tsutsui, Y. Endo, S. Sagawa, F. Yamazaki, and K. Shiraki, "Sympathetic nervous and hemodynamic responses to lower body negative pressure in hyperbaria in men," American Journal of Physiology-Regulatory, Integrative and Comparative Physiology, vol. 282, no. 1, pp. R38-R45, 2002.

[7] D. R. Seals, D. G. Johnson, and R. F. Fregosi, "Hyperoxia lowers sympathetic activity at rest but not during exercise in humans," American Journal of Physiology-Regulatory, Integrative and Comparative Physiology, vol. 260, no. 5, pp. R873-R878, 1991.
[8] H. Al Haddad, P. B. Laursen, D. Chollet, F. Lemaitre, S. Ahmaidi, and M. Buchheit, "Effect of cold or thermoneutral water immersion on post-exercise heart rate recovery and heart rate variability indices," Autonomic Neuroscience, vol. 156, no. 1-2, pp. 111-116, 2010.

[9] R. Perini and A. Veicsteinas, "Heart rate variability and autonomic activity at rest and during exercise in various physiological conditions," European journal of applied physiology, vol. 90, no. 3-4, pp. 317-325, 2003.

[10] J. Schipke and M. Pelzer, "Effect of immersion, submersion, and scuba diving on heart rate variability," British Journal of Sports Medicine, vol. 35, no. 3, pp. 174-180, 2001.

[11] F. Chouchou, V. Pichot, M. Garet, J.-C. Barthélémy, and F. Roche, "Dominance in cardiac parasympathetic activity during real recreational scuba diving," European journal of applied physiology, vol. 106 , no. 3, p. $345,2009$.

[12] L. Ueno, M. Miyachi, T. Matsui, K. Takahashi, K. Yamazaki, K. Hayashi, S. Onodera, and T. Moritani, "Effect of aging on carotid artery stiffness and baroreflex sensitivity during head-out water immersion in man," Brazilian journal of medical and biological research, vol. 38, no. 4, pp. 629-637, 2005.

[13] A. Hernando, M. Pelaez, M. T. L. Albalate, M. Aiger, D. Izquierdo, A. Sanchez, M. I. Lopez-Jurado, J. I. Moura, J. Fidalgo, J. Lazaro, et al., "Autonomic nervous system measurement in hyperbaric environments using ecg and ppg signals," IEEE Journal of Biomedical and Health Informatics, 2018.

[14] M. L. Westerlund, "Classification with kohonen self-organizing maps," Soft Computing, Haskoli Islands, 2005.

[15] L. Fang, H. Zhao, P. Wang, M. Yu, J. Yan, W. Cheng, and P. Chen, "Feature selection method based on mutual information and class separability for dimension reduction in multidimensional time series for clinical data," Biomedical Signal Processing and Control, vol. 21, pp. 82-89, 2015.

[16] Y. Noh, H. F. Posada-Quintero, Y. Bai, J. White, J. P. Florian, P. R. Brink, and K. H. Chon, "Effect of shallow and deep scuba dives on heart rate variability," Frontiers in physiology, vol. 9, p. 110, 2018.

[17] S. Kujawski, J. Słomko, M. Zawadka-Kunikowska, M. Kozakiewicz, J. J. Klawe, M. Tafil-Klawe, and P. Zalewski, "Effects of hyperbaric exposure on the cardiovascular system. role of the autonomous nervous system," Polish Hyperbaric Research, vol. 61, no. 4, pp. 33-42, 2017. 